\title{
A Metamaterial-Inspired Structure for Simultaneous Vibration Attenuation and Energy Harvesting
}

\author{
Winner Anigbogu' ${ }^{1}$ and Hamzeh Bardaweel $\mathbb{D}^{1,2,3}$ \\ ${ }^{1}$ Institute for Micromanufacturing, College of Engineering and Science, Louisiana Tech University, Ruston, LA 71272, USA \\ ${ }^{2}$ Department of Mechanical Engineering, College of Engineering and Science, Louisiana Tech University, Ruston, LA 71272, USA \\ ${ }^{3}$ Department of Nanosystems Engineering, College of Engineering and Science, Louisiana Tech University, Ruston, LA 71272, USA
}

Correspondence should be addressed to Hamzeh Bardaweel; hamzehb@latech.edu

Received 8 January 2020; Revised 15 May 2020; Accepted 20 May 2020; Published 13 June 2020

Academic Editor: C. M. Wang

Copyright (c) 2020 Winner Anigbogu and Hamzeh Bardaweel. This is an open access article distributed under the Creative Commons Attribution License, which permits unrestricted use, distribution, and reproduction in any medium, provided the original work is properly cited.

\begin{abstract}
In this article, a magnetomechanical metamaterial structure capable of simultaneous vibration attenuation and energy harvesting is presented. The structure consists of periodically arranged local resonators combining cantilever beams and permanent magnetcoil systems. A prototype of the metamaterial dual-function structure is fabricated, and models are developed. Results show good agreement between model simulation and experiment. Two frequency bandgaps are measured: $205-257 \mathrm{~Hz}$ and $587-639 \mathrm{~Hz}$. Within these bandgaps, vibrations are completely attenuated. The level of vibration attenuation in the first bandgap is substantially larger than the level of vibration attenuation observed in the second bandgap. Mode shapes suggest that bending deformations experienced by the local resonators in the second bandgap are less than the deformations experienced in the first bandgap, and most vibrational energy is localized within the first bandgap where the fundamental resonant frequency is located, i.e., $224 \mathrm{~Hz}$. The ability of the fabricated metamaterial structure to harvest electric power in these bandgaps is examined. Results show that vibration attenuation and energy harvesting characteristics of the metamaterial structure are coupled. Stronger vibration attenuation within the first bandgap has led to enhanced energy harvesting capabilities within this bandgap. Power measurements at optimum load resistance of $15 \Omega$ reveal that maximum power generated within the first bandgap reaches $5.2 \mu \mathrm{W}$ at $245 \mathrm{~Hz}$. Compared with state-of-the-art, the metamaterial structure presented here shows a significant improvement in electric power generation, at considerably lower load resistance, while maintaining the ability to attenuate undesired vibrations within the frequency bandgap.
\end{abstract}

\section{Introduction}

Elastic mechanical metamaterials have shown extraordinary properties including bandgap phenomenon [1-3]. Consequently, elastic metamaterials are gaining unprecedented attention in advanced engineering applications such as vibration attenuation and wave filtering [4-6] and energy harvesting $[7,8]$. Typically, the overall mechanical metamaterial structure is man-made and consists of periodically arranged cavities housing locally resonating masses that are connected to the cavities by elastic elements, i.e., springs [9]. The bandgap phenomenon is the result of the dynamic behavior of the periodically arranged local resonators and Bragg's scattering in periodic structures. The overall dynamics of the metamaterial structure is determined by the locally resonating masses. When the mechanical metamaterial structure is subject to excitations from external source, the kinetic energy contained in these oscillations is trapped in the local resonators. This results in the unique capability of generating frequency bandgaps within which oscillations are prevented from passing through. The generated bandgaps are formed at frequency matching the fundamental resonant frequency of the local resonators that are acting as local absorbers within the metamaterial structure $[2,10]$.

To this end, research interests in mechanical metamaterials have grown in a few directions including: vibration attenuation and wave filtering, vibration energy 
harvesting, and simultaneous vibration attenuation and energy harvesting. For instance, Matlack et al. presented an elastic metastructure for broadband vibration absorption [5]. The structure was additively manufactured using fused deposition-modeling technique and then assembled manually in order to insert metallic cubes inside the printed structure. Moreover, a metamaterial structure embedded with hierarchically organized local resonators was fabricated and characterized by Xu et al. [11]. Both experimental and numerical methods were used to fully characterize the fabricated structure. Results from this study demonstrated the ability of the fabricated structure to attenuate vibrations within two bandgaps in frequency regions of $144-188 \mathrm{~Hz}$ and $480-810 \mathrm{~Hz}$. In a similar fashion, Zhu et al. built a chiral lattice-based metamaterial beam that is embedded with local resonators to achieve broadband vibration attenuation in the frequency range of, approximately, $210-700 \mathrm{~Hz}$ [12].

The use of metamaterial structures to generate electric power via energy harvesting has also been investigated recently. A thorough review of metamaterials-based energy harvesters was presented by Chen et al. [7]. For example, a metamaterial energy harvester employing piezoelectrics was fabricated [13]. The harvester was shown to produce peak power in the order to $100 \mu \mathrm{W}-1$ $\mathrm{mW}$ at $55 \mathrm{kHz}$ and $30-80 \mathrm{k} \Omega$. Concurrent vibration attenuation and energy harvesting metamaterials have been recently, also, demonstrated. Chen et al. built a metamaterial beam embedded with periodic cells that consist of membrane-split-ring resonators [14]. PVDF piezoelectric patch was attached to the membranes to convert the kinetic energy captured by the resonators into useful electric power. The fabricated structure exhibited simultaneous vibration attenuation and energy harvesting capabilities. Excellent and moderate vibration attenuation characteristics were reported in two bandgaps, i.e., $340-426 \mathrm{~Hz}$ and $460-500 \mathrm{~Hz}$, respectively. The fabricated structure recovered approximately $0.5 \mu \mathrm{W}$ across $200 \mathrm{k} \Omega$ at $348 \mathrm{~Hz}$. Power was only measured and reported within the first bandgap, i.e., at $348 \mathrm{~Hz}$. Also, a recent study by $\mathrm{Li}$ et al. reported a mechanical metamaterial structure for simultaneous vibration isolation and energy harvesting [15]. The structure consisted of beams with PVDF piezoelectric transducers to extract electric energy from the resonating cells. The metamaterial structure was able to attenuate vibrations and generate electric power, simultaneously, within $146-171 \mathrm{~Hz}$ frequency bandgap. Specifically, a peak power of $0.05 \mu \mathrm{W}$ was measured across $1 \mathrm{M} \Omega$ within the frequency bandgap.

The current state-of-the-art literature reveals that a growing number of researchers have recently attempted to use elastic mechanical metamaterials for simultaneous vibration attenuation and energy harvesting. The work in this area is still new and rapidly growing [16]. Therefore, the work we present in this article is focused on investigating a magnetomechanical-based metamaterial structure for simultaneous vibration attenuation and energy harvesting. The presented structure consists of a combination of cantilever beams and permanent magnet-coil systems in order to achieve two main functions, simultaneously. These functions are vibration attenuation and energy harvesting. Compared with other efforts and available literature, the metamaterial structure we present in this work is shown to generate, significantly, more electric power while maintaining its ability to attenuate undesired vibrations. Additionally, the presented structure requires lower load resistance to achieve optimum power transfer, compared with what has been presented in the literature [13-15]. This is an important feature of the presented structure because, typically, electronic circuits for sensors require an input current in the order of $10 \mathrm{~mA}$ [17]. As the load resistance increases the deliverable electric current decreases. It is desirable to achieve maximum power transfer at the lowest load resistance values [17]. The rest of the article is organized as follows: Section 2 presents the aspects of the proposed design and fabrication methodology of the metamaterial structure. Section 3 deals with modeling of the metamaterial structure. Section 4 is focused on characterization tests and experimental methods. Findings and results are presented and discussed in Section 5.

\section{Design and Fabrication}

Figure 1 displays the design and concept of the metamaterial vibration attenuation energy harvesting system presented in this work. The metamaterial structure, shown in Figure 1(a), consists of local resonators (unit cells). Each unit cell, shown in Figure 1(b), contains four angled cantilevers with fixed-free ends. A permanent magnet is placed in the center of each unit cell. The tip mass at the free end of each cantilever is made of copper coils wrapped around the free end of the cantilever as shown in Figure 1(c). The coils serve two purposes: first, the coils form additional mass to lower the resonant frequency of the vibrating cantilevers, thus lowering the frequency bandgap of the metamaterial structure; second, the magnet-coil system is used to extract electric energy from kinetic energy of the resonating cantilevers.

When the metamaterial structure is subject to external vibrations, the vibrational energy is trapped into the local resonators, causing the cantilevers to resonate. That is, when the driving frequency of vibration matches the resonant frequency of the local resonators, the vibration energy is transferred into kinetic energy using the resonating cantilevers, and therefore, this energy is localized inside the resonators. This results in frequency bandgaps. Consequently, these undesired vibrations are blocked from traveling across the ends of the metamaterial structure. Thus, the first function of the proposed metamaterial vibration attenuation energy harvesting system is achieved. Concurrently, by integrating the coils and permanent magnets into the design of the local resonators, as shown in Figure 1(c), the kinetic energy of the resonating cantilevers is converted into useful electric power. Subsequently, voltage is induced in the coils wrapped around the free end 


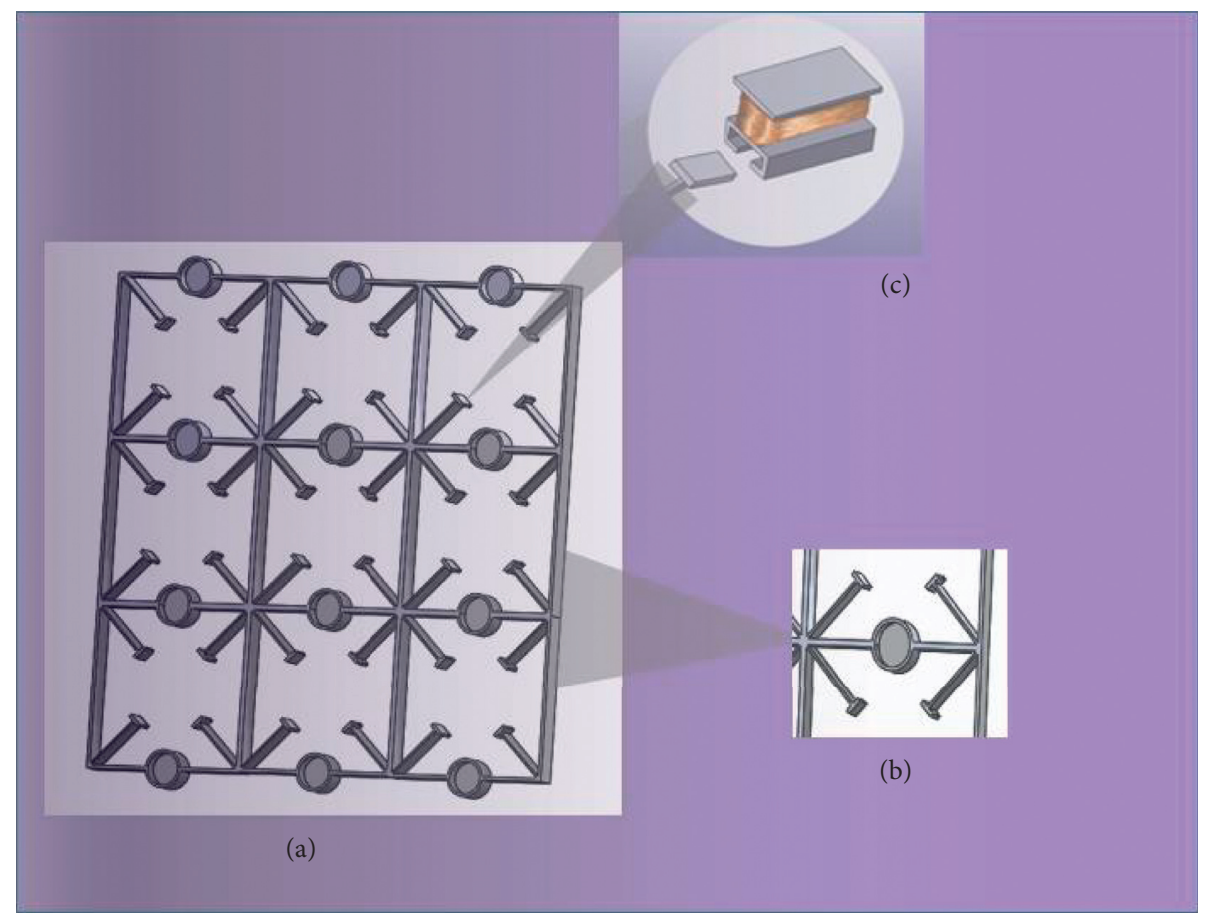

Figure 1: Concept and design of the metamaterial vibration attenuation energy harvesting structure presented in this work: (a) design layout of the metamaterial structure; (b) building block, i.e., local resonator. Each local resonator consists of four angled cantilevers and a central magnet mass, and (c) the tip of each cantilever is made of insertion where copper coils are placed for power extraction.

of the cantilevers. Therefore, the second function of the proposed system is achieved by converting the kinetic energy contained in these vibrations into useful electric power.

\section{Theory}

Theoretical models of the proposed metamaterial vibration attenuation energy harvesting structure are developed next. First, COMSOL FEM software is used to investigate frequency bandgaps and modes of vibrations. Second, a theoretical model is developed using first principle and used to investigate wave attenuation capabilities of the presented metamaterial structure.

3.1. COMSOL FE Model. Figure 2 shows the geometries and dimensions of the cantilever, i.e., the building block of the metamaterial structure presented in this work. First, a $3 \mathrm{D}$ CAD drawing of the unit cell was prepared using SolidWorks software, and the predetermined geometries and dimensions are shown in Figure 2. The CAD drawing was then imported into COMSOL finite-element multiphysics software to analyze the dynamic response of the metamaterial structure and obtain the dispersion diagram that depicts the relationship between the wave number, $\mathscr{K}$, and frequencies for an infinite number of periodically arranged unit cells. That is, for an array of unit cells, at frequency intervals that meet Bragg's scattering condition, destructive interference occurs. This yields the desired frequency bandgap. The presence of the local resonators in the structure further enhances the frequency bandgap because, at the resonant frequency of the locally resonating structures, the kinetic energy contained in the external vibrations is transferred to the local resonators, causing them to resonate while creating a lower frequency bandgap in the dispersion diagram of the metamaterial structure.

In the COMSOL model, a fine mesh coupled with the choice of material properties (given in Table 1) and geometries (shown in Figure 2) is used to simulate the dispersion curve and obtain frequency bandgaps. The model assumes no damping effects and solves the governing equation of the metamaterial structure given by

$$
\begin{aligned}
\mathbf{K}_{d} & =\left[\begin{array}{ll}
\mathbf{K}_{\mathbf{u}}-\omega^{2} \mathbf{M}_{\mathbf{u}}
\end{array}\right], \\
{\left[\begin{array}{lll}
\mathbf{K}_{\mathbf{u}}-\omega^{2} \mathbf{M}_{\mathbf{u}}
\end{array}\right]\left[\begin{array}{lll}
\mathbf{q}_{\mathbf{L}} & \mathbf{q}_{\text {int }} & \mathbf{q}_{\mathbf{R}}
\end{array}\right]^{T} } & =\left[\begin{array}{lll}
\mathbf{F}_{\mathbf{L}} & 0 & \mathbf{F}_{\mathbf{R}}
\end{array}\right]^{T},
\end{aligned}
$$

where $\omega$ and $\mathbf{K}_{\mathbf{d}}$ are frequency and dynamic stiffness matrix of the unit cell, respectively, $\mathbf{K}_{\mathbf{u}}$ and $\mathbf{M}_{\mathbf{u}}$ are stiffness and mass matrices of the unit cell, respectively. Here, $\mathbf{q}_{\mathbf{L}}, \mathbf{q}_{\mathbf{R}}$, and $\mathbf{q}_{\text {int }}$ are displacement vector of the left side of the unit cell, displacement vector of the right side of the unit cell, and displacement vector of the internal nodes of the unit cell, respectively. Similarly, $\mathbf{F}_{L}$ and $\mathbf{F}_{R}$ are force vectors on the left and right sides of the unit cell, respectively. Upon finite element discretization, the unit cell contained 11,647 elements with a maximum size of $1.81 \mathrm{~mm}$ and a minimum size of $0.0775 \mathrm{~mm}$.

To solve the governing equation of the system, standard Bloch-Floquet theory is imposed on the displacement, $\mathbf{q}$, and force, $\mathbf{F}$, vectors of each node, thus taking care of the periodicity condition of the unit cells given by 


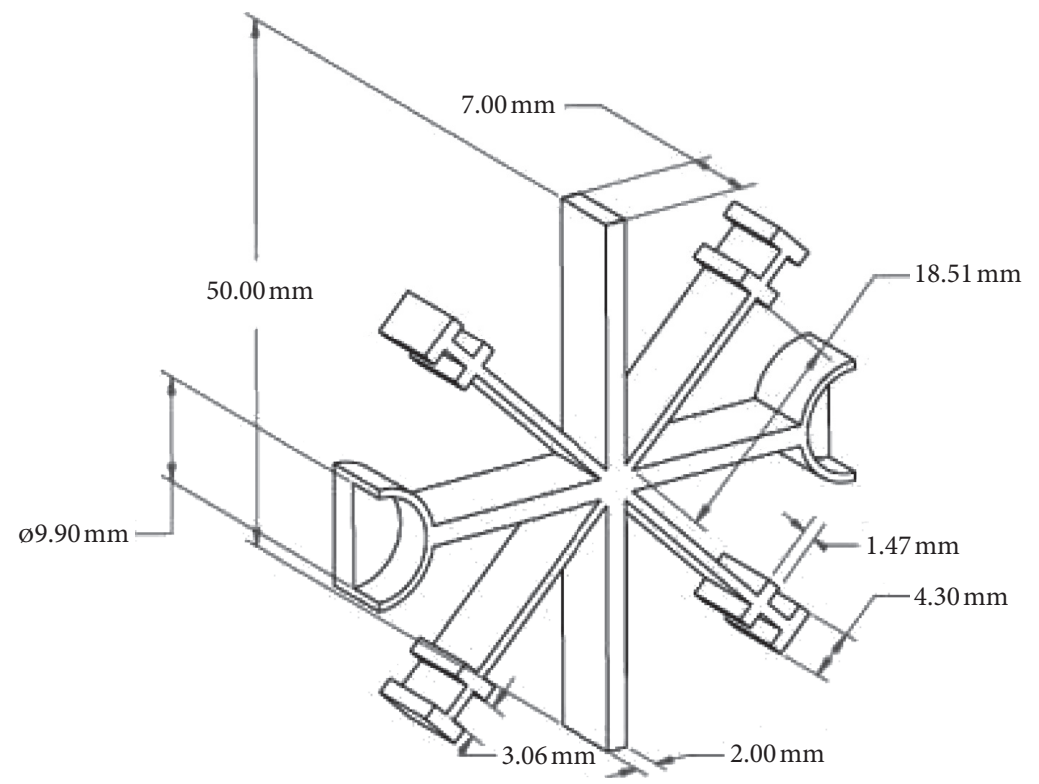

FIGURE 2: Metamaterial cantilever beams and central magnet used in model simulations.

Table 1: Properties and dimensions of the metamaterial vibration attenuation energy harvesting structure used in model simulations.

\begin{tabular}{lc}
\hline Unit cells & \\
\# of cantilever beams & 4 per unit cell \\
\# of central magnets & 1 per unit cell \\
\# of unit cells & 9 \\
\hline Coils & \\
Type and grade & AW \\
\# Turns & 220 \\
Length & $9.04 \mathrm{~m}$ \\
\hline Central magnets & NdFeB-N52 \\
Material & $9.525 \mathrm{~mm}$ \\
Diameter, $D_{m}$ & $4.7625 \mathrm{~mm}$ \\
Thickness, $H_{m}$ & \\
\hline Cantilever & PETG \\
Material & $1270 \mathrm{Kg} / \mathrm{m}^{3}$ \\
Density, $\rho$ & $2.7 \mathrm{GPa}$ \\
Modulus of elasticity, $E$ & 0.33 \\
Poisson's ratio, $v$ & $18.51 \mathrm{~mm}$ \\
Length, $L$ & $7 \mathrm{~mm}$ \\
Width, $W$ & $1 \mathrm{~mm}$ \\
Height, $H$ & $745 \mathrm{~N} / \mathrm{m}^{-4}$ \\
Stiffness, $k$ & $1.65 \times 10^{-4} \mathrm{~kg}$ \\
Mass of cantilever, $m_{\text {can }}$ & $0.00127 \mathrm{~kg}$ \\
mass, $m$ & $1.4 \times 10^{-5} \mathrm{~m}^{2}$ \\
Cross-sectional area, $A$ & $4.6667 \times 10^{-12} \mathrm{~m}^{4}$ \\
Area moment of inertia, $I$ &
\end{tabular}

$$
\begin{aligned}
& \mathbf{q}_{\mathbf{R}}=-e^{i k l_{u}} \mathbf{q}_{\mathbf{L}}, \\
& \mathbf{F}_{\mathbf{R}}=-e^{i k l_{u}} \mathbf{F}_{\mathbf{L}},
\end{aligned}
$$

where $l_{u}=50 \mathrm{~mm}$ is the length of the unit cell as shown in Figure 2. Next, the described eigenvalue problem is solved for a given wave number in order to obtain the corresponding Eigen frequencies. A band structure is obtained by sweeping the wave vector along the irreducible Brillouin zone. This results in dispersion curves defining frequency bandgaps where oscillations are blocked and prevented from passing through. The tolerance was set to $1 \times 10^{-6}$.

3.2. Analytical Model. The metamaterial vibration attenuation energy harvester structure is represented as Euler-Bernoulli beam with periodic arrangement of local resonators attached to the beam. This is shown in Figure 3. Each local resonator consists of a cantilever with linear stiffness coefficient, $k$, and mass, $m$. In this work, transfer matrix method is used in modeling the wave propagation across the beam, assuming small displacement of the beam [18].

The general equation of motion of the Euler-Bernoulli beam is given as

$$
\mathrm{EI} \frac{\partial^{4} y}{\partial x^{4}}+\rho A \frac{\partial^{2} y}{\partial t^{2}}=0,
$$

where $y(x, t)$ is the dynamic deflection of the neutral axis at point $x, E$ is the elastic modulus of the beam, $I$ is the area moment of inertia, $\rho$ is the density of the beam, and $A$ is the cross-sectional area. In this work, it is assumed that $y(x, t)=Y(x)^{i \omega t}$, where $Y(x)$ defines the mode shapes at point $x$, described as

$$
Y(x)=Q \cos (\gamma x)+B \sin (\gamma x)+C \cosh (\gamma x)+D \sinh (\gamma x),
$$

where $\gamma$ is the flexural wave number given as

$$
\gamma^{4}=\frac{\rho A \omega^{2}}{\mathrm{EI}} .
$$

For the $n_{\text {th }}$ unit cell, the mode shape function can be rewritten as

$$
Y_{n}\left(x^{\prime}\right)=Q_{n} \cos \left(\gamma x^{\prime}\right)+B_{n} \sin \left(\gamma x^{\prime}\right)+C_{n} \cosh \left(\gamma x^{\prime}\right)+D_{n} \sinh \left(\gamma x^{\prime}\right),
$$




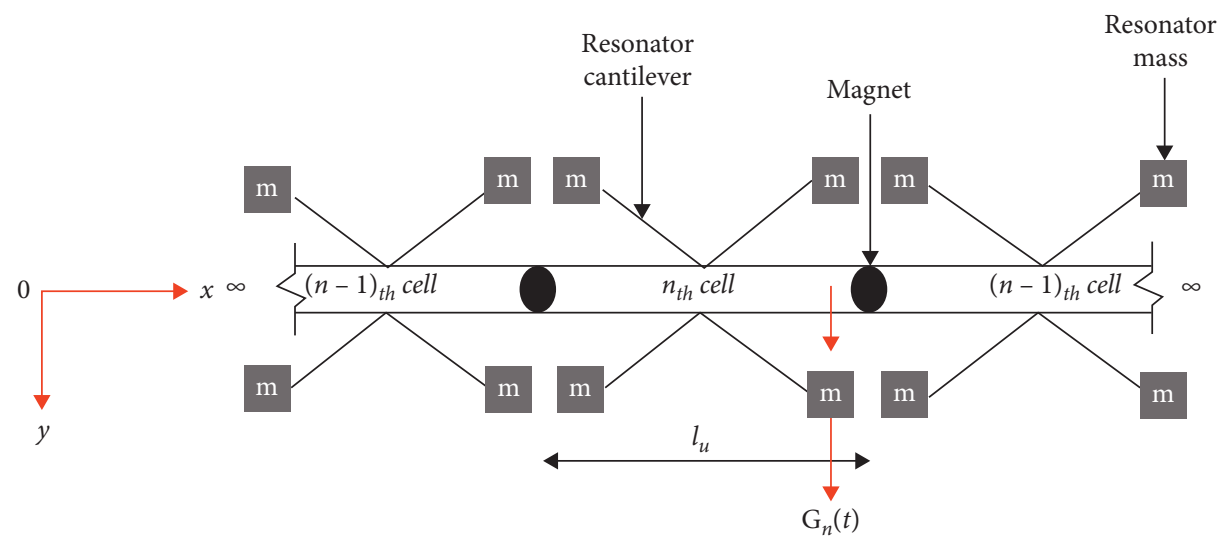

Figure 3: Cartoon model schematic of beam with local resonators representing the metamaterial vibration attenuation energy harvesting structure.

where $\quad x^{\prime}=x-n l_{u}, \quad n l_{u} \leq x \leq(n+1) l_{u}$. Here, $Q_{n}, B_{n}$, $C_{n}$, and $D_{n}$ are the unknown amplitudes describing the mode shape function [19].

3.3. Analysis of the Local Resonator (LR). The equation of the $n_{\text {th }}$ local resonator in Figure 3 is described as

$$
F\left(x_{n}, t\right)+m \ddot{G}_{n}(t)=0,
$$

where $F\left(x_{n}, t\right)$ is the force of the $n_{\text {th }}$ resonator developed as a result of interaction with the beam at the contact point as shown in Figure 3 and $G_{n}$ is the vertical displacement of the mass of the resonator, $m$, during the external excitation. The force, $F\left(x_{n}, t\right)$, is given by

$$
F\left(x_{n}, t\right)=-k\left[y\left(x_{n}, t\right)-G_{n}(t)\right],
$$

where the spring stiffness coefficient, $k$, of the local resonator cantilever is given as $k=E W H^{3} / 4 L^{3}$, where $W$ and $H$ are the width and height of the cantilever, and $L$ is the overall length of the cantilever.

Assuming the solution for the vertical displacement of the mass resonator of the form:

$$
G_{n}(t)=V_{n} e^{i \omega t},
$$

where $V_{n}$ is the displacement of the local resonator (LR), and combining equations (7)-(9) yields the following equation:

$$
m \ddot{G}_{n}=k\left[y\left(x_{n}, t\right)-G_{n}(t)\right] .
$$

Substituting $G_{n}$ and $y\left(x_{n}, t\right)$ into equation (10) gives

$$
-m \omega^{2} V_{n} e^{i \omega t}=k\left[Y_{n}(0)-V_{n}\right] e^{i \omega t} .
$$

Rearranging equation (11), the vertical displacement, $V_{n}$, can be written as

$$
V_{n}=\frac{k Y_{n}(0)}{\left(k-m \omega^{2}\right)} .
$$

Moreover, equation (8) can be rewritten as

$$
F\left(x_{n}, t\right)=-k\left[Y_{n}(0)-V_{n}\right] e^{i \omega t},
$$

and substituting equation (12) into equation (13) yields

$$
F\left(x_{n}, t\right)=\frac{m \omega^{2} k}{\left(k-m \omega^{2}\right)} Y_{n}(0) e^{i \omega t}=F_{n} e^{i \omega t} .
$$

The model for the four unconnected resonators $(r=$ $1,2,3,4)$ shown in Figure 3, at the interface with the beam can be derived as [20]

$$
m \ddot{G}_{n, r}=k_{r}\left[y\left(x_{n}, t\right)-G_{n, r}(t)\right], \quad r=1,2,3,4,
$$

with the amplitude of displacement of the local resonators, $V_{n}$, given by

$$
V_{n, r}=\frac{k_{r} Y_{n}(0)}{\left(k_{r}-m_{r} \omega^{2}\right)} \quad r=1,2,3,4
$$

Consequently, the force acting on the beam due to these four resonators can be obtained from equations (13) and (14) and is given by:

$$
\begin{aligned}
F\left(x_{n}, t\right) & =\sum-\left(k_{r}\left[Y_{n}(0)-V_{n, r}\right] e^{i \omega t}\right. \\
& =\sum \frac{m_{r} \omega^{2} k_{r}}{\left(k_{r}-m_{r} \omega^{2}\right)} Y_{n}(0) e^{i \omega t}=F_{n} e^{i \omega t}, \quad r=1,2,3,4 .
\end{aligned}
$$

Next, the continuity conditions at the point where the resonators meet the beam, as shown in Figure 3, are imposed between the $n_{t h}$ unit cell and the $(\mathrm{n}-1)_{t h}$ cell. These include displacement, slope, bending moment, and shear force and, respectively, given by:

$$
\begin{aligned}
Y_{n-1}\left(l_{u}\right) & =Y_{n}(0), \\
Y_{n-1}^{\prime}\left(l_{u}\right) & =Y_{n}^{\prime}(0), \\
E I Y_{n-1}^{\prime \prime}\left(l_{u}\right) & =E I Y_{n}^{\prime \prime}(0), \\
E I Y_{n-1}^{\prime \prime \prime}\left(l_{u}\right) & =E I Y_{n}^{\prime \prime \prime}(0)-F_{n} .
\end{aligned}
$$



yields

Substituting equations (6) and (17) into equation (18) where $\psi_{\mathbf{n}}=\left(Q_{n}, B_{n}, C_{n}, D_{n}\right)^{T}$. Here, $\mathbf{U}$ and $\mathscr{M}$ are given by

$$
\psi_{\mathbf{n}}=\mathscr{M}^{-1} \mathbf{U} \psi_{\mathbf{n}-1}
$$

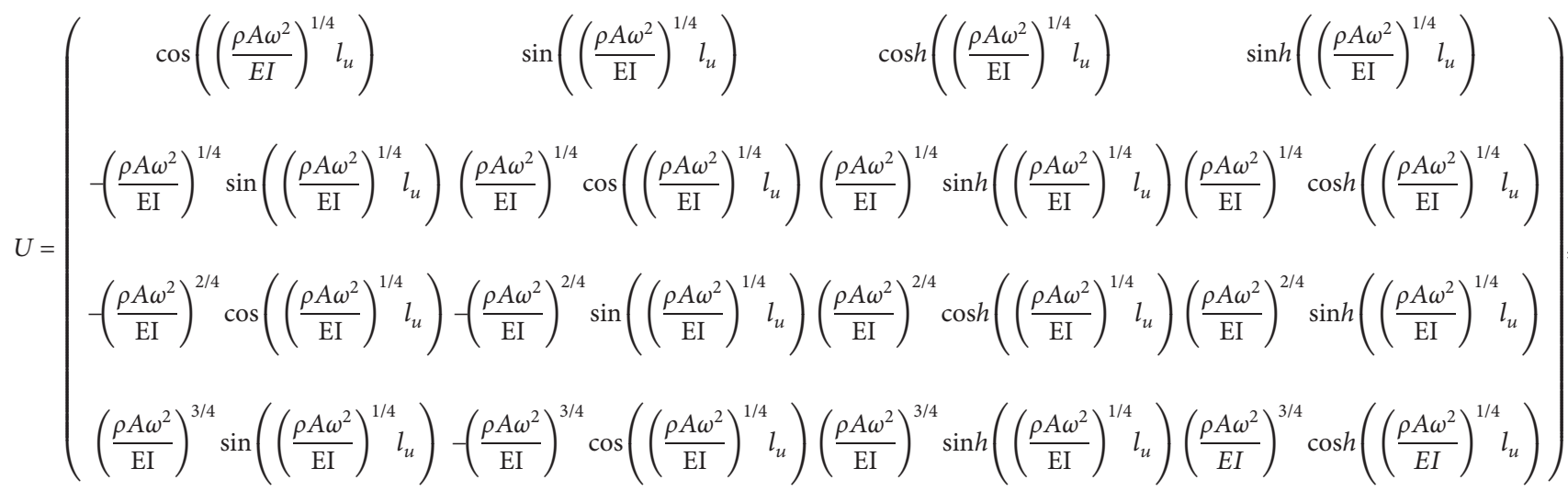

$$
\begin{aligned}
& \mathscr{M}=\left(\begin{array}{cccc}
1 & 0 & 1 & 0 \\
0 & \left(\frac{\rho A \omega^{2}}{\mathrm{EI}}\right)^{1 / 4} & 0 & \left(\frac{\rho A \omega^{2}}{\mathrm{EI}}\right)^{1 / 4} \\
-\left(\frac{\rho A \omega^{2}}{\mathrm{EI}}\right)^{2 / 4} & 0 & \left(\frac{\rho A \omega^{2}}{\mathrm{EI}}\right)^{2 / 4} & 0 \\
-F & -\left(\frac{\rho A \omega^{2}}{\mathrm{EI}}\right)^{3 / 4} & -F & \left(\frac{\rho A \omega^{2}}{\mathrm{EI}}\right)^{3 / 4}
\end{array}\right),
\end{aligned}
$$

respectively, and

$$
F=\frac{1}{\mathrm{EI}}\left(\sum \frac{m_{r} \omega^{2} k_{r}}{\left(k_{r}-m_{r} \omega^{2}\right)}\right), \quad r=1,2,3,4 .
$$

From Bloch-Floquet's equation of period structures in the $x$ direction, we have

$$
\psi_{\mathbf{n}}=e^{i z l_{u}} \psi_{\mathbf{n}-1}
$$

Further, by letting $\mathscr{M}^{-1} \mathbf{U}=\mathbf{S}$ and substituting equation (19) into equation (22) yield

$$
\mathbf{S} \psi_{\mathbf{n}-1}=e^{i z l_{u}} \psi_{\mathbf{n}-1}
$$

Equation (23) can be further rearranged into a standard eigenvalue problem described by

$$
\left|\mathbf{S}-e^{i z l_{u}} \mathbf{I}\right|=0
$$

where $\mathbf{I}$ is a $[4 \times 4]$ identity matrix.

\section{Experimental Methods}

A prototype of the metamaterial vibration attenuation energy harvesting structure was built and characterized experimentally. The fabrication process involved two main steps. First, the CAD design of the metamaterial structure was prepared using SolidWorks software and then converted into STL (STereoLithography) format in order to perform $3 \mathrm{D}$ printing of the prototype. The Ultimaker 3 Fused Deposition Modeling (3D FDM) printer was used to build the metamaterial structure using PETG (polyethylene terephthalate) filament. Second, 35 AWG enameled copper wires were winded around the free end of the cantilevers and neodymium iron boron $(\mathrm{NdFeB})$ permanent solid magnets were manually integrated into the $3 \mathrm{D}$ printed metamaterial structure. Properties and dimensions of the fully assembled metamaterial vibration attenuation energy harvesting structure are given in Table 1 . Figure 4 shows the fully fabricated and assembled metamaterial vibration attenuation energy harvester structure prototype.

The experiment apparatus used to characterize the response of the metamaterial vibration attenuation energy harvester prototype is shown in Figure 5. The apparatus consists of a shaker table (VT-500, SENTEK DYNAMICS), power amplifier (LA-800, SENTEK DYNAMICS), vibration controller (S81B-P02, SENTEK DYNAMICS), accelerometers (PCB333B30 model, PCB Piezotronics), decade box (Global Specialties RDB-10), and PC. Characterization tests were focused on, simultaneously, measuring vibration transmissibility and the amount of electric power recovered 


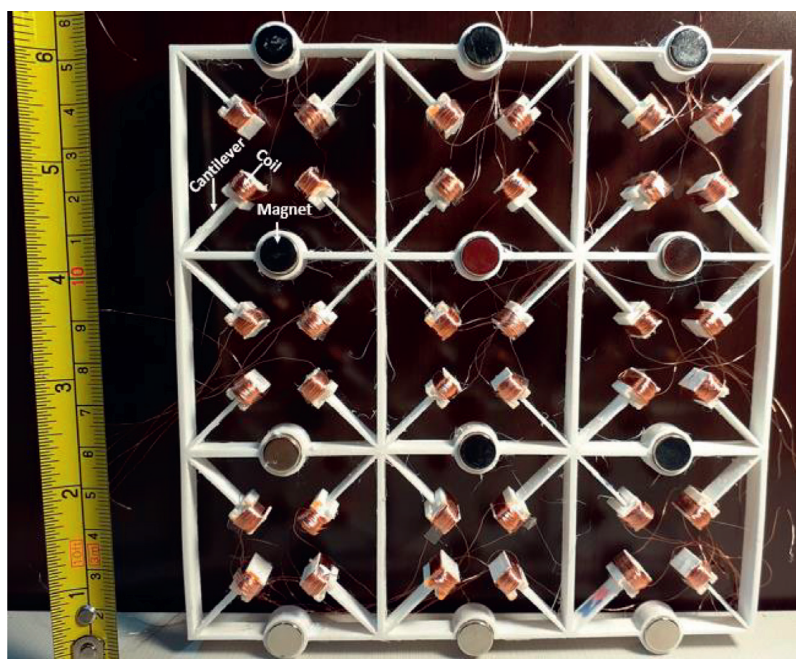

Figure 4: Prototype of the metamaterial vibration attenuation energy harvesting structure presented in this work.

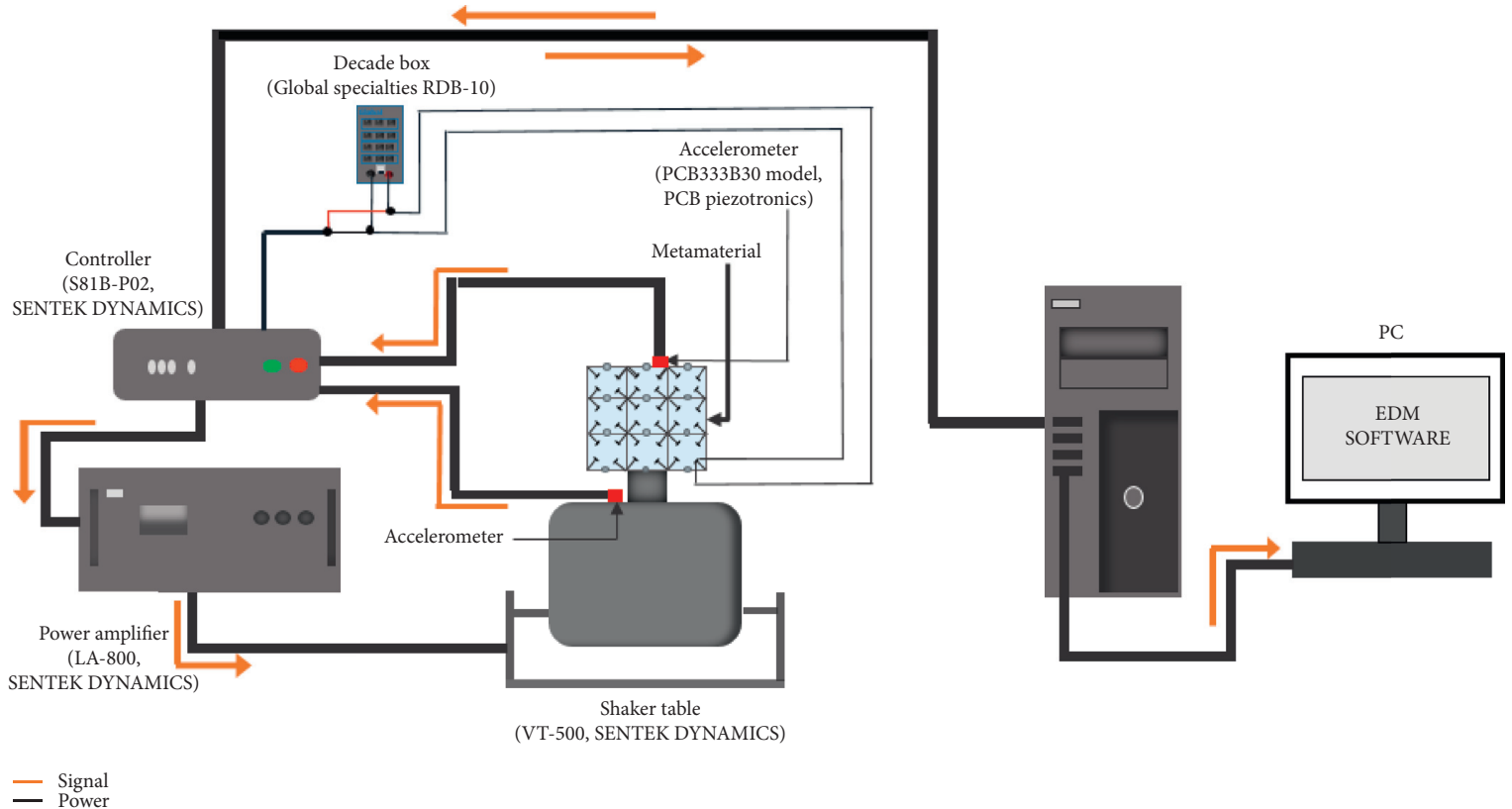

FIGURE 5: Cartoon schematic of the experimental apparatus used for characterization of the fabricated metamaterial vibration attenuation energy harvesting structure.

from a unit cell. The electromagnetic shaker table was used to excite the metamaterial structure harmonically at predetermined frequencies and acceleration levels. An accelerometer was fixed, on top of the shaker table, and used to monitor the input waveform and acceleration level delivered to the metamaterial structure. When performing these experiments, the lower end of the metamaterial vibration attenuation energy harvester was secured on top of the shaker table as shown in Figure 5. A fixture and clamps were used to constrain and fix the top end of the metamaterial structure (not shown in Figure 5). A second accelerometer was fixed on the top end of the metamaterial structure and used to measure the vibration transmissibility of the metamaterial structure. Both input and output signals from the top and bottom accelerometers were measured and stored on the PC to analyze the vibration transmissibility. That is, the vibration transmissibility was measured by tracking the level of acceleration transmitted from the metamaterial structure relative to the input acceleration from the shaker table. Concurrently, the output voltage from a unit cell was measured across a load resistance and used to estimate the amount of recovered electric power.

\section{Results and Discussion}

First, we start by investigating wave propagation through the metamaterial structure. Using equation (24), for every selected frequency value, $\omega$, the solutions of the wave vector, $z$, 


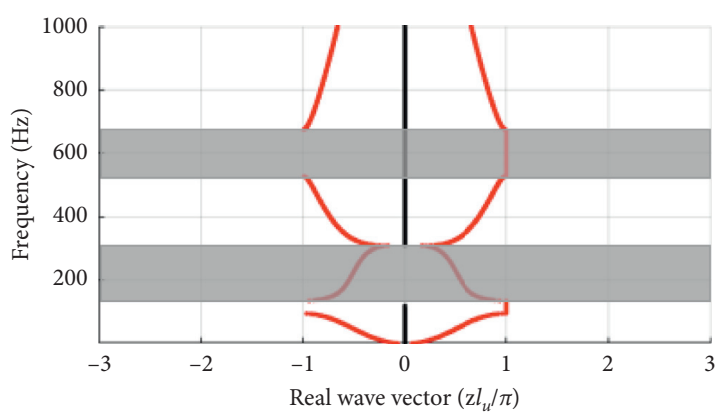

FIGURE 6: Band structure obtained by solving equation (24) to investigate the wave attenuation capabilities of the metamaterial structure. First and second frequency bandgaps are $121-310 \mathrm{~Hz}$ (lower frequency range) and 528-674 Hz (higher frequency range), respectively (marked in gray).

are obtained. Figure 6 shows the results obtained from solving the eigenvalue problem given in equation (24). Here, a stopband exists when $z$ is imaginary, while a pass-band exists when $z$ is real [19]. As shown in Figure 6, the stopbands of the wave are marked in gray. Results reveal that there exist two main stopbands in the frequency range $1-1000 \mathrm{~Hz}$. These bands are occurring in the range of $121-310 \mathrm{~Hz}$ (lower frequency range) and $528-674 \mathrm{~Hz}$ (higher frequency range), respectively. These two bands are the result of the beam itself and the local resonators (LR) [21]. That is, a higher frequency stopband is created as a result of vibration phase differences-in-phase and out-ofphase-between adjacent unit cells. A low-frequency stopband is typically generated by the LR stopband due to the attenuation effect of the resonators.

Next, to fully understand the behavior of the presented metamaterial vibration attenuation energy harvesting structure, we further analyze the structure using COMSOL FEM model described in Section 3.1 and experiment described in Section 4. The frequency bandgaps of the metamaterial vibration attenuation energy harvesting structure shown in Figure 7 are analyzed using COMSOL FEM. Periodic boundary conditions were imposed, and geometric and material properties were set similar to those measured and reported in Table 1. Figure 7 shows the dispersion curve of the metamaterial structure obtained using COMSOL model described in Section 3. Figure 7 suggests that there exists two major bandgaps within the frequency range of $100-1000 \mathrm{~Hz}$. The first and second bandgaps are $218-247 \mathrm{~Hz}$ and $589-780 \mathrm{~Hz}$, respectively.

To further explore these bandgaps, Figure 8 shows the corresponding mode shapes obtained using COMSOL at the lower and upper edges of the first and second bandgaps, i.e., $218,247,589$, and $780 \mathrm{~Hz}$, respectively. Clearly, within these bandgaps, vibrations are attenuated and trapped within the locally resonating cantilevers. Also, the fundamental resonant frequency of the unit cell (combined cantilever beams, tip coils, and central magnet) was estimated using COMSOL at approximately $224 \mathrm{~Hz}$. The fundamental resonant frequency falls within the first bandgap of the metamaterial structure, i.e., 218-247 Hz. As shown in Figure 8, at the lower and upper edges of the first bandgap, the deformations of the

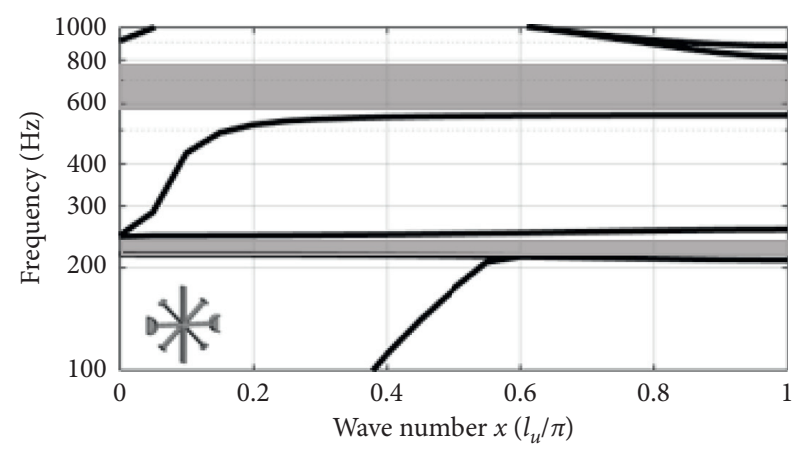

FIGURE 7: Dispersion curve and frequency bandgaps of a metamaterial structure consisting of cantilever beams with tip coils and central magnet obtained using COMSOL simulations. Frequency bandgaps: the first and second bandgaps are $218-247 \mathrm{~Hz}$ and $589-780 \mathrm{~Hz}$, respectively (marked in gray).

unit cell are localized within the cantilevers. The vibrational energy is localized within the frequency bandgap and converted into kinetic energy of the resonating cantilevers. Also, Figure 8 suggests that maximum bending deformations occur within the first bandgap. Therefore, it is expected that most of energy harvesting will occur within the first bandgap. This observation agrees with the vibration energy harvesting measurements that were performed in this work and discussed later in this article. Since most mechanical vibrations in nature occur at frequencies lower than $300 \mathrm{~Hz}$ [15], in this work, energy harvesting is targeted within this bandgap, i.e., $218-247 \mathrm{~Hz}$.

Next, vibration transmissibility of the fabricated metamaterial structure was measured using experiment apparatus shown in Figure 5. In these experiments, a sinusoidal vibration sweep was performed in the range of $50-1000 \mathrm{~Hz}$ at an acceleration level of $0.5 \mathrm{~g} \mathrm{~m} . \mathrm{s}^{-2}$. Figure 9 shows the measured vibration transmissibility of the metamaterial structure versus frequency. Clearly, the results from this experiment demonstrate two frequency bandgaps (marked in red). Results from COMSOL model simulations and analytical model (equation (24)) are displayed in Table 2. Results show a good agreement between models' predictions and measured data for both bandgaps. However, some discrepancy between models' predictions and measured data is evident. For example, the measured bandwidth of the first bandgap $(205-257 \mathrm{~Hz})$ is wider than the bandwidth of the COMSOL simulation-based first bandgap. A similar observation was made and reported by Chen et al. [14]. This may be attributed to some of the approximations made in the model simulations including absence of damping [14] or other experimental variations such as insignificant distortion in the metamaterial structure during the experiment. Similarly, the first bandgap obtained from equation (24) is a little wider than the bandgap predicted by COMSOL simulations and experiment as shown in Table 2. This is likely because of some of the simplifications and approximations made in the model (equation (24)) including absence of damping. Also, the analytical model does not factor in some of the design complexities including the flange attachment at the contact point with the beam. The model also assumes 


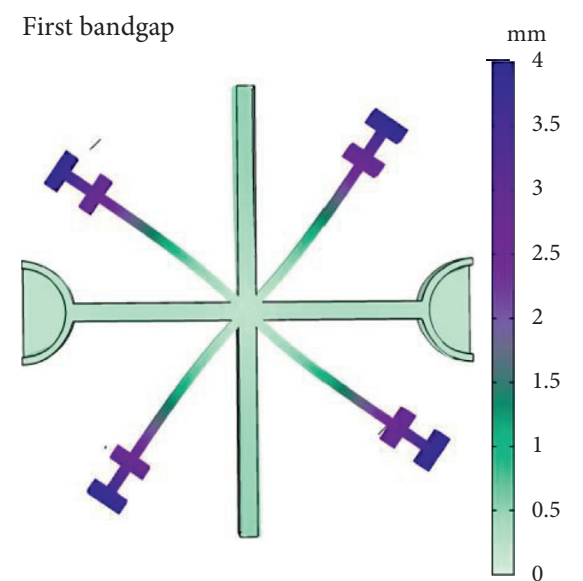

$218 \mathrm{~Hz}$

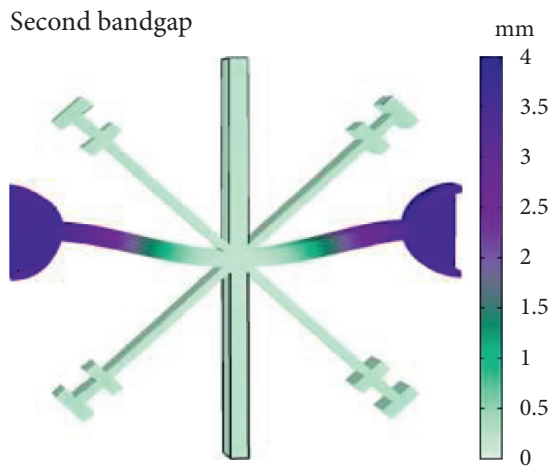

$589 \mathrm{~Hz}$

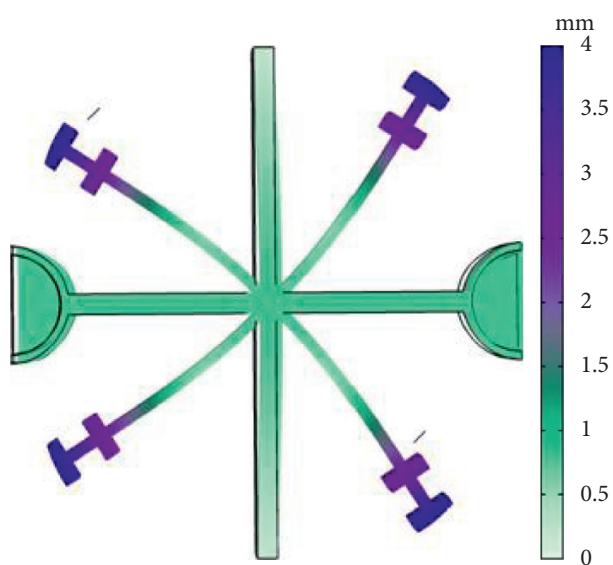

$247 \mathrm{~Hz}$

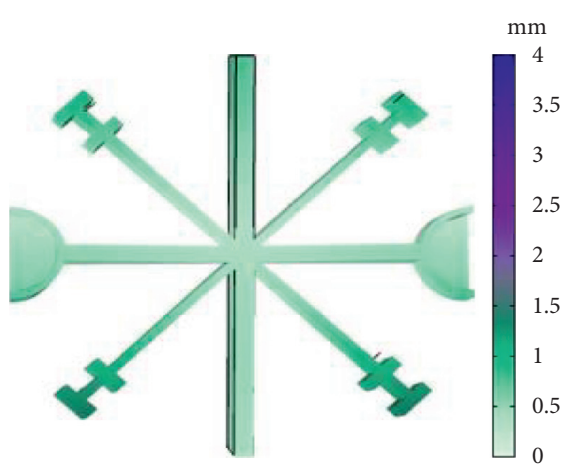

$780 \mathrm{~Hz}$

FIgURE 8: Mode shapes at the edge frequencies of each bandgap obtained using COMSOL model simulations.

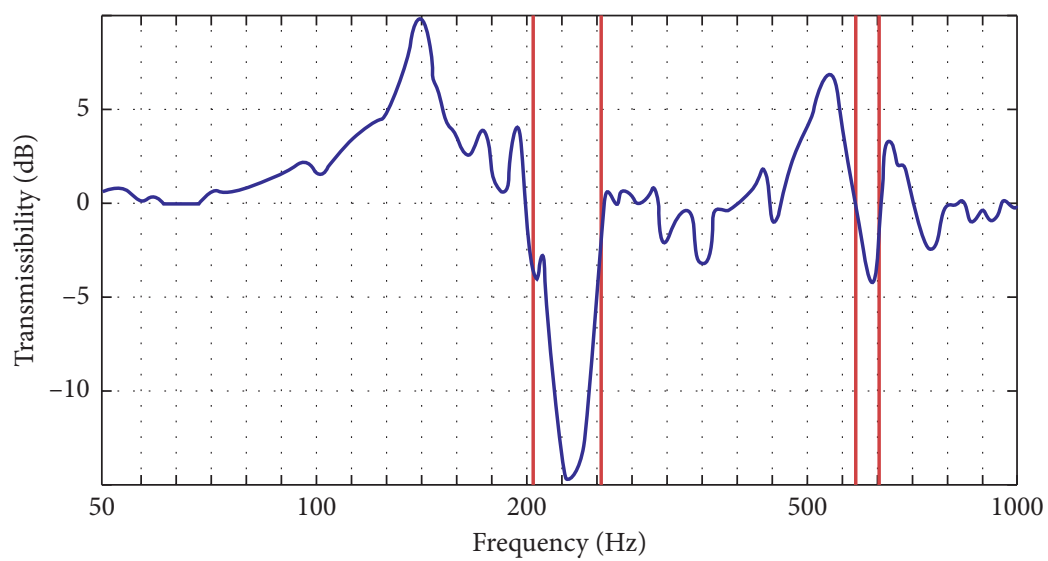

FIGURE 9: Vibration transmissibility versus frequency for the metamaterial structure obtained using experiment. Measured frequency bandgaps (marked in red) are shown.

small displacements of the transverse beam and ignores the mass contribution from the transverse beam. Nonetheless, both models predict similar behaviors and show similar trends compared with measured data. Furthermore, results from experiment show that the level of vibration attenuation, represented as the dip in the transmissibility curve in Figure 9, occurring in the first bandgap is substantially larger than the level of vibration attenuation observed in the second bandgap. This behavior is expected since, as discussed earlier and shown in Figure 8, the second bandgap experiences less deformations compared with the first bandgap, and most vibrational energy is localized within the first bandgap where the fundamental resonant frequency is located, i.e., $224 \mathrm{~Hz}$.

The ability of the fabricated metamaterial structure to scavenge electric power while concurrently attenuating undesired vibrations is examined next. Figure 10 shows electric power harvested from one cantilever located in the 
TABLE 2: Comparison between frequency bandgaps obtained using the experiment and models.

\begin{tabular}{lccc}
\hline & Experiment & COMSOL simulations & Equation (24) \\
\hline First band $(\mathrm{Hz})$ & $205-257$ & $218-247$ & $121-310$ \\
Second band $(\mathrm{Hz})$ & $587-639$ & $589-780$ & $528-674$ \\
\hline
\end{tabular}

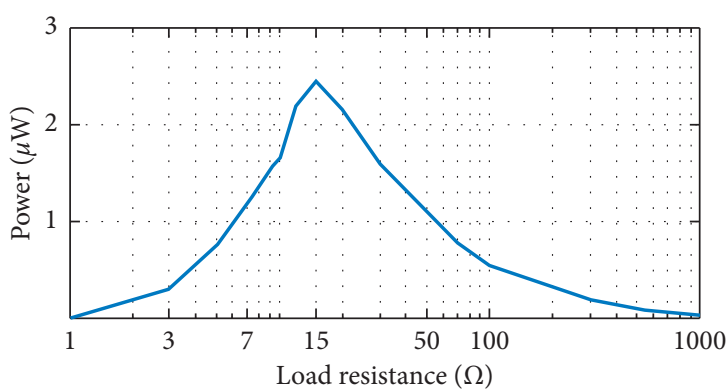

(a)

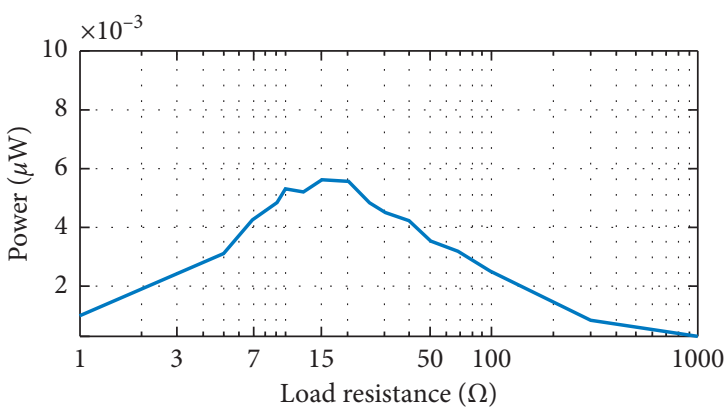

(b)

Figure 10: Measured electric power from a single cantilever within a unit cell at (a) $223 \mathrm{~Hz}$ and (b) $615 \mathrm{~Hz}$ and $0.5 \mathrm{~g}$ m.s .

bottom central unit cell of the metamaterial structure. In this experiment, the metamaterial structure was driven harmonically at $0.5 \mathrm{~g} \cdot \mathrm{m} . \mathrm{s}^{-2}$ and fixed frequency (selected within the first bandgap, i.e., $223 \mathrm{~Hz}$ ) while the load resistance was swept in the range of 1-1000 $\Omega$ (Figure 10(a)). The same experiment was repeated at a fixed frequency selected from the second bandgap, i.e., $615 \mathrm{~Hz}$, as shown in Figure 10(b). Results from these experiments confirm that most of the vibrational energy is localized within the first bandgap, causing the cantilevers to locally resonate. Consequently, within the first bandgap, kinetic energy in these resonating cantilevers is effectively converted into electric power through the coils at the free end of these cantilevers. Maximum power outputs of, approximately, $2.5 \mu \mathrm{W}$ and $0.6 \mathrm{nW}$, were measured at $223 \mathrm{~Hz}$ (first bandgap) and $615 \mathrm{~Hz}$ (second bandgap), respectively. Thus, results demonstrate that both vibration transmissibility (Figure 9) and energy harvesting (Figure 10) characteristics of the fabricated metamaterial structure are more prominent in the first frequency bandgap. Also, Figure 10 reveals that the maximum power output occurs at optimum load resistance of $15 \Omega$.

It is also worth noting that the improvement in energy harvesting within the first bandgap $(205-257 \mathrm{~Hz})$ is accompanied by stronger vibration attenuation. That is, vibration attenuation and energy harvesting characteristics of the metamaterial structure are coupled. Stronger vibration attenuation leads to enhanced vibration energy harvesting capabilities. This is further demonstrated in Figure 11 which shows the output electric power versus frequency. In this experiment, the metamaterial structure was swept harmonically at $0.5 \mathrm{~g} \mathrm{~m} . \mathrm{s}^{-2}$ in the frequency range of $50-500 \mathrm{~Hz}$. The load resistance was fixed at the optimum value, i.e., $15 \Omega$, in order to maximize power generation. It is clear that, within the bandgap $(205 \mathrm{~Hz}-257 \mathrm{~Hz})$, the large dip in the transmissibility curve (Figure 9) corresponds to the maximum power generation (Figure 11). Also, Figure 11 reveals that the maximum power generated within the frequency bandgap can reach up to, approximately, $5.2 \mu \mathrm{W}$ at $245 \mathrm{~Hz}$.

Next, performance metrics of the presented metamaterial vibration attenuation energy harvesting structure are compared to the state-of-the-art work reported in the recent literature. This is shown in Table 3. The fabricated metamaterial vibration attenuation energy harvesting structure performed very well compared with other efforts recently reported in the literature. For example, the metamaterial structure was able to attenuate undesired vibrations at the, typically, targeted frequencies, i.e., less than $300 \mathrm{~Hz}$. Concurrently, the presented metamaterial structure produced significantly more electric power compared with what has been reported in the literature. Table 3 also shows another advantage of the work presented in this article. The optimum load resistance to maximize electric power was significantly lower compared with what has been reported in the literature. This low impedance guarantees higher electric current that is desirable to operate electronic circuits for sensors, as discussed earlier in this article. Although our initial prototyping efforts produced a metamaterial structure with comparable metrics to what has been reported in the literature, it should be noted that optimization of this metamaterial dual-purpose structure is beyond the scope of work reported in this article. The work reported in this article is focused on design, proof-of-concept experiments, and characterization tests of the metamaterial structure rather than optimization. Several design improvements can be made to improve the overall performance of the fabricated metamaterial dual-function structure. This includes optimization of geometries and dimensions of the cantilever beams, tilt angle of cantilever beams, number of unit cells, and size and location of central magnets, etc. This will require developing a detailed and sophisticated coupled model of the magnetomechanical metamaterial vibration attenuation energy harvesting structure and, therefore, will be the focus of future work. 


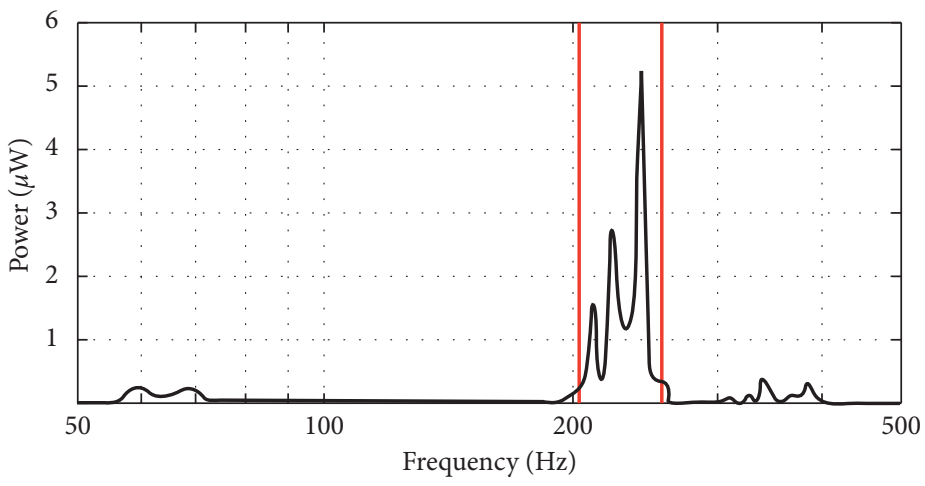

Figure 11: Measured output electric power spectrum from a single cantilever at fixed, optimum, load resistance of $15 \Omega$ and acceleration level of $0.5 \mathrm{~g} \mathrm{~m} . \mathrm{s}^{-2}$. Measured frequency bandgap is denoted in red.

Table 3: Comparison between the presented work and recent advancements in the field.

\begin{tabular}{|c|c|c|c|c|}
\hline & $\begin{array}{l}\text { Load resistance } \\
\qquad(\mathrm{K} \Omega)\end{array}$ & $\begin{array}{c}\text { Power } \\
\text { output }(\mu \mathrm{W})\end{array}$ & $\begin{array}{l}\text { First frequency } \\
\text { bandgap }(\mathrm{Hz})\end{array}$ & Specifications \\
\hline $\begin{array}{l}\text { Ying Li et al. } \\
\text { [15] }\end{array}$ & 1,000 & 0.05 & $146-171$ & $\begin{array}{c}\text { Simultaneous vibration attenuation and energy harvesting using } \\
\text { piezoelectric, PVDF. }\end{array}$ \\
\hline $\begin{array}{l}\text { Jung-San Chen } \\
\text { et al. [14] }\end{array}$ & 200 & 0.5 & $340-426$ & $\begin{array}{c}\text { Simultaneous vibration attenuation and energy harvesting using } \\
\text { piezoelectric, PVDF. }\end{array}$ \\
\hline $\mathrm{Xu}$ et al. [11] & - & - & $144-188$ & Only vibration attenuation. \\
\hline Zhu et al. [12] & - & - & $210-700$ & Only vibration attenuation. \\
\hline $\begin{array}{l}\text { Kyung Ho Sun } \\
\text { et al. [22] }\end{array}$ & 20 & 0.345 & - & $\begin{array}{l}\text { Only energy harvesting capability using acoustic metamaterial and a } \\
\text { double-clamped piezoelectric bimorph plate. Results were reported } \\
\text { at } 600 \mathrm{~Hz} .\end{array}$ \\
\hline This work & 0.015 & 5.2 & $205-257$ & $\begin{array}{c}\text { Simultaneous vibration attenuation and energy harvesting using } \\
\text { magnet-coil system. }\end{array}$ \\
\hline
\end{tabular}

\section{Conclusions}

The work presented in this article is focused on the design and characterization of a magnetomechanical-based metamaterial structure for simultaneous vibration attenuation and energy harvesting. The structure consists of periodically arranged unit cells that are made of a combination of cantilever beams and permanent magnet-coil systems. Each unit cell contains four angled cantilevers with fixed-free ends. A permanent magnet is placed in the center of each unit cell. The tip mass at the free end of each cantilever is made of copper coils that serve two purposes: lowering the frequency bandgap of the metamaterial structure and extracting electric energy from kinetic energy of the resonating cantilevers as they move near the central permanent magnet. A prototype of the metamaterial dual-function structure has been fabricated using additive manufacturing and then assembled manually. Models of the structure have been developed and validated against the experiment.

Results show good agreement between model simulations and experiment. Two frequency bandgaps, i.e., $205-257 \mathrm{~Hz}$ and $587-639 \mathrm{~Hz}$, respectively, have been measured. Within these two bandgaps, vibrations are completely attenuated. Results suggest that the level of vibration attenuation in the first bandgap is substantially larger than the level of vibration attenuation observed in the second bandgap. Mode shapes obtained using COMSOL FEM model reveal that the second bandgap is accompanied by less bending deformations compared with the first bandgap and most vibrational energy is localized within the first bandgap where the fundamental resonant frequency is located, i.e., $224 \mathrm{~Hz}$. Moreover, the ability of the fabricated metamaterial structure to harvest electric power while synchronously attenuating undesired vibrations has been inspected. Electric power outputs of, approximately, $2.5 \mu \mathrm{W}$ and $0.6 \mathrm{nW}$ were measured at $223 \mathrm{~Hz}$ (first bandgap) and $615 \mathrm{~Hz}$ (second bandgap) at optimum load resistance of $15 \Omega$. These results confirm that most of the vibrational energy is localized within the first bandgap, causing the cantilevers to locally resonate and kinetic energy in these resonating cantilevers to be, effectively, converted into useful electric power through the coils at the free ends of these cantilevers. Results also show that vibration attenuation and energy harvesting characteristics of the metamaterial structure are coupled. Stronger vibration attenuation has led to enhanced vibration energy harvesting capabilities. Frequency sweep measurements at optimum load resistance of $15 \Omega$ reveal that maximum power generated within the first frequency bandgap reaches, approximately, $5.2 \mu \mathrm{W}$ at $245 \mathrm{~Hz}$.

Compared with the state-of-the-art and reported literature, the metamaterial structure we present in this work has shown an order of magnitude improvement in electric power generation, at substantially lower optimum load resistance, while maintaining the ability to attenuate undesired 
vibrations in a frequency bandgap of $205-257 \mathrm{~Hz}$. Future work will focus on optimization of the metamaterial structure to maximize energy harvesting while maintaining vibration attenuation capabilities.

\section{Data Availability}

Data reported in this article are available from the corresponding author upon request.

\section{Conflicts of Interest}

The authors declare that they have no conflicts of interest regarding the publication of this article.

\section{Authors' Contributions}

Winner Anigbogu was involved in the data collection, formal analysis, software, validation, investigation, visualization, and formal analysis. Hamzeh Bardaweel was responsible for the conceptualization, funding acquisition, methodology, project administration, resources, software supervision, validation, visualization, and writing, reviewing, and editing of the original draft.

\section{Acknowledgments}

This study was partially supported by the Louisiana Board of Regents Support Fund (LEQSF(2015-18)-LaSPACE).

\section{References}

[1] G. Hu, L. Tang, and R. Das, "Metamaterial-inspired piezoelectric system with dual functionalities: energy harvesting and vibration suppression," in Active and Passive Smart Structures and Integrated Systems 2017, International Society for Optics and Photonics, Bellingham, WA, USA, 2017.

[2] Z. Wang, Q. Zhang, K. Zhang, and G. Hu, "Tunable digital metamaterial for broadband vibration isolation at low frequency," Advanced Materials, vol. 28, no. 44, pp. 9857-9861, 2016.

[3] O. Abdeljaber, O. Avci, S. Kiranyaz, and D. J. Inman, "Optimization of linear zigzag insert metastructures for lowfrequency vibration attenuation using genetic algorithms," Mechanical Systems and Signal Processing, vol. 84, pp. 625641, 2017.

[4] O. Casablanca, G. Ventura, F. Garescì et al., "Seismic isolation of buildings using composite foundations based on metamaterials," Journal of Applied Physics, vol. 123, no. 17, p. 174903, 2018.

[5] K. H. Matlack, A. Bauhofer, S. Krödel, A. Palermo, and C. Daraio, "Composite 3D-printed metastructures for lowfrequency and broadband vibration absorption," Proceedings of the National Academy of Sciences, vol. 113, no. 30, pp. 8386-8390, 2016.

[6] K. K. Reichl and D. J. Inman, "Lumped mass model of a 1D metastructure for vibration suppression with no additional mass," Journal of Sound and Vibration, vol. 403, pp. 75-89, 2017.

[7] Z. Chen, B. Guo, Y. Yang, and C. Cheng, "Metamaterialsbased enhanced energy harvesting: a review," Physica B: Condensed Matter, vol. 438, pp. 1-8, 2014 Apr 1.
[8] M. Saadatzi, F. Mir, M. N. Saadatzi, and S. Banerjee, "Modeling and fabrication of a multi-axial piezoelectric energy harvester based on a metamaterial-inspired structure," IEEE Sensors Journal, vol. 18, no. 22, pp. 9410-9419, 2018.

[9] M. Nouh, O. Aldraihem, and A. Baz, "Vibration characteristics of metamaterial beams with periodic local resonances," Journal of Vibration and Acoustics, vol. 136, Article ID 061012, 2014.

[10] T. Jiang and Q. He, "Dual-directionally tunable metamaterial for low-frequency vibration isolation," Applied Physics Letters, vol. 110, Article ID 021907, 2017.

[11] X. Xu, M. V. Barnhart, X. Li, Y. Chen, and G. Huang, "Tailoring vibration suppression bands with hierarchical metamaterials containing local resonators," Journal of Sound and Vibration, vol. 442, pp. 237-248, 2019.

[12] R. Zhu, X. N. Liu, G. K. Hu, C. T. Sun, and G. L. Huang, “A chiral elastic metamaterial beam for broadband vibration suppression," Journal of Sound and Vibration, vol. 333, no. 10, pp. 2759-2773, 2014.

[13] M. Carrara, M. R. Cacan, J. Toussaint, M. J. Leamy, M. Ruzzene, and A. Erturk, "Metamaterial-inspired structures and concepts for elastoacoustic wave energy harvesting," Smart Materials and Structures, vol. 22, no. 6, Article ID 065004, 2013.

[14] J.-S. Chen, W.-J. Su, Y. Cheng, W.-C. Li, and C.-Y. Lin, “A metamaterial structure capable of wave attenuation and concurrent energy harvesting," Journal of Intelligent Material Systems and Structures, vol. 30, no. 20, pp. 2973-2981, 2019.

[15] Y. Li, E. Baker, T. Reissman, C. Sun, and W. K. Liu, "Design of mechanical metamaterials for simultaneous vibration isolation and energy harvesting," Applied Physics Letters, vol. 111, no. 25 , p. 251903, 2017.

[16] G. Hu, L. Tang, A. Banerjee, and R. Das, "Metastructure with piezoelectric element for simultaneous vibration suppression and energy harvesting," Journal of Vibration and Acoustics, vol. 139, no. 1, 2017.

[17] M. Gao, Y. Wang, Y. Wang, and P. Wang, "Experimental investigation of non-linear multi-stable electromagnetic-induction energy harvesting mechanism by magnetic levitation oscillation," Applied Energy, vol. 220, pp. 856-875, 2018.

[18] L. Liu and M. I. Hussein, "Wave motion in periodic flexural beams and characterization of the transition between Bragg scattering and local resonance," Journal of Applied Mechanics, vol. 79, 2012.

[19] J. Zhou, K. Wang, D. Xu, and H. Ouyang, "Local resonator with high-static-low-dynamic stiffness for lowering band gaps of flexural wave in beams," Journal of Applied Physics, vol. 121, Article ID 044902, 2017.

[20] Z. Wang, P. Zhang, and Y. Zhang, "Locally resonant band gaps in flexural vibrations of a Timoshenko beam with periodically attached multioscillators," Mathematical Problems in Engineering, vol. 2013, Article ID 146975, 10 pages, 2013.

[21] M. Y. Wang and X. Wang, "Frequency band structure of locally resonant periodic flexural beams suspended with force-moment resonators," Journal of Physics D: Applied Physics, vol. 46, no. 25, p. 255502, 2013.

[22] K. H. Sun, J. E. Kim, J. Kim, and K. Song, "Sound energy harvesting using a doubly coiled-up acoustic metamaterial cavity," Smart Materials and Structures, vol. 26, Article ID 075011, 2017. 